\title{
Understanding Embryonic Stem Cell Pluripotency Through Its Key Players
}

\author{
Rekha Govindan ${ }^{\#}$ \\ ${ }^{\#}$ School of Biotechnology, National Institute of Technology, NIT Campus (P.O), Calicut - 673601
}

\begin{abstract}
Certain transcription factors, cofactors, chromatin regulators are identified as the key players in establishing the embryonic stem cell ground state. The activation and silencing of genes in response to potency and lineage development act as the regulatory circuits. Insights into these regulatory circuitory have uncovered fundamental genetic and epigenetic mechanisms that control gene expressions to maintain the ground state. At one end of the circuit are the core transcription factors that self regulate their own gene expression. These factors further activate transcription of genes involved in pluripotency and contribute to the arrest of expression of lineage specific genes. The core transcription factors mediate binding of the enhancers with signaling factors there by extending the circuit to communicate the signals directly to the genes regulated by the core factors. The core factors also create an undifferentiated state through chromatin regulators that remodify the regulatory gene sequences of specific lineages to inactive states. Understanding the embryonic stem cell ground state in terms of its various regulatory aspects serves as both a baseline for understanding the changes that occur as cells differentiate and develop and as a means to understand the basic biology of these cells. It also helps in understanding the mechanisms behind induced pluripotent cells in vitro culture conditions that might contribute to stem cell based translational medicines.
\end{abstract}

Key Words: Stem cells, ground state, gene circuits, signaling molecules, epigenetic influences

\section{Introduction}

Stem cell research is a relatively new technology that takes primitive human cells that are called as stem cells and develops them into most any of the 220 varieties of cells in the human body, including blood cells and brain cells. In culture conditions, these cells can be propagated in an undifferentiated state but can be induced to differentiate into specialized cell types (Thomson et al.,2011; Zeineddine et al.,2014). Self renewal is the ability to produce more stem cells under appropriate culture conditions. Pluripotency is the intrinsic ability of a stem cell in which even after prolonged expansion in culture, they retain full responsiveness to developmental cues and can differentiate into any type of somatic lineages or germline cells upon reintroduction to the embryo. In induced pluripotent stem cells, the pluripotency is generated in vitro by reprogramming germ cells with nuclear transfer (SCNT) or somatic cells by introducing extrinsic factors through gene transfection techniques. This vital aspect of pluripotent stem cells makes them indispensable tools for developmental biology, disease modeling and regenerative medicine (Boyer et al.,2005; Takahashi and Yamanaka, 2006; Silva and Smith, 2008; Kerr et al.,2008; Zhao et al.,2012). The critical features of any stem cell, whether of embryonic, fetal or adult origin, is its developmental potential, defined operationally as the range of precursor or mature tissue cell types the stem cell can produce in a given experimental context. Embryogenesis is generally viewed as a series of committed events, during which the developmental capacity of the cells of the embryo becomes gradually restricted (Fig.1), so that once the tissues of the body have been formed, their constitutive stem cells can only give rise to a restricted range of cell types (Silva and Smith, 2008; Nichols and Smith, 2012; Zhu and Huangfu, 2013).

\section{Stemness of ESCs - the ground state}

Pluripotency or stemness is defined as the ability of a cell to differentiate into all of the cell types of an organism. It is a characteristic of cells in the inner cell mass (ICM) of the preimplantation blastocyst which are considered to be the embryonic stem cells. In vivo, as the development proceeds, pluripotency is lost from the differentiating cells implying the nature of pluripotency a transient state. However, embryonic stem cells (ESCs) derived from the ICM of the blastocyst retains pluripotency indefinitely in vitro. This is considered to be the ground state of the embryonic stem cells where the cells though undifferentiated, upon culture induction, can be differentiated into specific lineages (Jaenisch and Young, 2008; Lee et al.,2010; Thomson et al.,2011). Induced pluripotent stem cells (iPSCs) have been derived by using cocktails of certain extrinsic factors. Barring slight changes at the histone modifications, a similarity in the developmental potency and gene expression were noted between ESCs and iPSCs. Such an observation draws a highly significant context as iPSCs might represent a state where by extrinsic addition of factors reestablish the ESC ground state as well as the ESC regulatory circuitry (Takshashi and Yaamanaka, 2006; Yamanaka and Blau, 2010; Young, 2011). In embryonic stem (ES) 
cells and iPSCs, events leading to the maintenance of pluripotency and reacquisition of stemness by somatic reprogramming represent distinct processes that however act through a set of similar transcriptional events underlining the pluripotent state. In both ES and iPS cells, the fundamental transcription frameworks are governed by a common set of "core" stem cell-specific transcription factors, namely Oct4, Sox2, and Nanog (Fig.2). These major three activators, in turn complex with both ubiquitous and cell type-specific transcription factors to orchestrate complex gene expression circuits that determines whether the stem cells should maintain the unique ability to safeguard stemness or as the signals changes, to execute gene expressions that induces lineage specification. The three players form a genetic circutory that is assisted by the coordinated recruitment of coactivator proteins to their cognate promoters. These coactivators brings upon genetic and epigenetic changes that initiates downstream activities once the circutory is constituted. Some of the coactivators are histone modifiers (e.g., $\mathrm{CBP} / \mathrm{p} 300$ ) and chromatin remodelers (e.g., PBAF/BAF) to gene promoters and alter chromatin structure towards unwinding state that is more accessible for binding of subsequent factors and transcriptional activation. A few activators are like molecular adaptors that bridge activators to the major key players Oct4, Sox2 and Nanog through protein-protein interactions to execute specific transcriptional programs. An overall synergistic mechanism is found throughout the circuitry system (Jaenisch and Young, 2008; Van den Berg et al.,2010; Pierantozzi et al.,2010; Fong et al.,2011; Han et al.,2012).

Within the embryonic stem cell, the gene circuits forms auto- and cross regulatory loops of core factors and downstream pluripotency / self-renewal genes. From the top of the circuit, the core transcription factors Oct4, Sox 2 and Nanog activate target genes encoding signaling components and other pluripotency factors. Through the cross regulatory loops, they control cell differentiation by repressing the expression of genes encoding components of pathways that promote ES cell differentiation. Downstream of the core factors in the circuit are the coactivators and enhancers that may further repress genes that induce ES cell differentiation, thereby reinforcing the ground state. The binding sites of Oct4, Sox2, and Nanog are in proximity such a way that they all bind to their own promoters, as well as the promoters of the genes encoding the two other factors. For eg., Oct 4 and Sox 2 co-occupy and activate the Oct 4 and Nanog genes. This enables the three factors to function collaboratively to maintain their own expression. Autoregulation is thought to enhance the stability of gene expression thereby the pluripotent state is indefinitely maintained. Such auto regulatory loops are a general feature of master regulators of cell state. Formation of an auto regulatory loop by Oct4, Sox 2 and Nanog serves to decide whether the cell has to choose either self renewal or differentiation. Oct4, Sox2, and Nanog co-occupy several hundred genes, often at apparently overlapping genomic recognition sequences. Such multiprotein complex containing Oct4 and Nanog can be derived by immunoprecipitation in pluripotent cells, suggesting evidence that multiple interacting proteins coordinately control pluripotency (Jaenisch and Young, 2008; Yamanaka and Blau, 2010; Young, 2011).

\section{Pluripotency as a platform for multilineage decisions where antagonistic gene circuits acts in creating a metastable ground state}

ES pluripotency can be considered as a consequence of interactions among various factors with the key players at the regulatory circuit. An uncommitted condition may be maintained if the gene regulatory networks within the ES cells are antagonistic in nature. This might takes place by restricting the probability of any single gene circuitory attaining an activation threshold for dominance over its key players Oct $4 / \mathrm{Sox} 2 / \mathrm{Nanog}$. The opposing transcription factor circuits might be neutralized with the creation of a pluripotent state. Analogous transcription factors might compete to establish multi lineage potentials in the ES cells. The Erk signal might destabilize the neutral status of the ES to promote lineage specification by upregulating or silencing selective gene circuits to enhance secondary inducers responsiveness to the downstream signaling pathway. Within the ES pluripotent circuit, Stat3 and ID proteins may directly antagonize effectors downstream of Erk while Nanog acts to inhibit transcriptional and epigenetic targets of lineage specification (Silva and Smith, 2008; Young, 2011).

Self renewal and pluripotency are the specific features of ES cell. The shift from one state to another might require factors that act antagonistically or when the gene circutory systems acts in tandem or in opposite. The interconnected gene circutory network formed by Oct 4 , Sox2, and Nanog generates a ground state and a transition state for ESCs. In the ground state, the factors are expressed at appropriate levels and pluripotency is maintained and in the transition state, due to non functional role of any one master player, the cell enters into the differentiation path (Boyer et al., 2005; Huang et al.,2007; Silva and Smith, 2008; Young, 2011). Oct4 is a protein transcription factor encoded by the gene Pou5f1. It is also known as Oct3, Oct3/4 and was first identified in mice as an ESC specific and germline specific transcription factor. Oct4 belongs to the POU transcription factor family, in which the members control the expression of their target genes through binding an octameric sequence motif of an AGTCAAAT consensus sequence. The importance of Oct4 was identified through the knockout of Oct4 that caused early lethality in mice and an improper ICM. Hence Oct4 is considered to play a pivotal role in the early mammalian development and in the self renewal of ES cells (Pan et al.,2002; Van den 
Berg et al.,2010; Lee et al.,2010; Tsai et al.,2012). Oct4 is known to interact with other transcription factors to activate and repress gene expression in mouse ES cells. Oct4 heterodimerizes with the HMG-box transcription factor Sox2. This dimeric association is thought to be the main mechanism for the developmental control of gene expression. Oct4 and Sox 2 were shown to interact synergistically to activate transcription of target genes and that this activity is dependent upon the correct juxtaposition of Oct4 and Sox 2 binding sites. Oct 4 and Sox 2 shared the promoter binding sites on the genes that were essential for the pluripotency. Nanog also showed an affinity to these binding sites. The sharing of binding sequence places the key factors to act together and simultaneously transactivate other genes and regulatory elements (Van den Berg et al.,2010; Lee et al.,2010; Tsai et al.,2012). Early levels of Oct4 expression is detected at the 4- to 8-cell stage. Thereafter, a high level of nuclear Oct4 protein is detected in all blastomeres until the morula stage. In the blastocyst, Oct4 expression remains high in the inner cell mass and is not expressed in the trophectoderm (TE). Once the embryo is implanted, transient upregulation of Oct4 in a group of cells of the ICM triggers their differentiation into primitive endoderm (hypoblast) cells. Subsequently, down regulation of Oct4 expression in these cells were observed. In the gastrula, Oct 4 expression is again down regulated and after day 8 of gestation, Oct 4 expression is confined to primordial germ cells. In vitro, Oct4 is highly expressed in undifferentiated embryonic stem (ES) cells, embryonic carcinoma (EC) cells and embryonic germ cells. Oct4 expression is downregulated with withdrawal of leukemia inhibitory factor (LIF). Retinoic acid is a potent repressor of Oct4 and presence of retinoic acid is found to induce loss of stemness and differentiation of cells into different lineages (Webster et al.,2007; Hammachi et al.,2011; Zeineddine et al.,2014). The Oct4 protein structure comprises three domains, a central POU (Pit-Oct-Unc) domain for DNA binding, an N-terminal transactivation domain and a C-terminal domain, which appears to be a cell type-specific transactivation domain. The upstream regulatory elements of Oct4 loci consist of a proximal enhancer and a distal enhancer, in addition to TATA less proximal promoter. Interestingly, the proximal enhancer and the distal enhancer function at different developmental stages: the proximal enhancer functions in the epiblast and epiblast stem cells, while the distal enhancer in the ICM, primordial germ cells and ESCs (Shi and Jin, 2010; Hammachi et al.,2011; Zeineddine et al.,2014). Oct4 is considered a master regulator for the initiation and maintenance of pluripotent cells during embryonic development. Oct4 mainly functions through the activation of pluripotency associated and self-renewalassociated genes, while simultaneously repressing genes that promote differentiation, in coordination with other pluripotency factors and coregulators. Several well-known Oct4 target genes include Fgf4, Utf1, Osteopontin, Rex1, Hand 1 and a and $\beta$ human chorionic gonadotropin (hCG). The target genes identified frequently encode transcription factors, many of which are developmentally important homeodomain proteins. The core Oct4 regulatory network is formed by these associated target genes and cofactors and other DNA binding elements. The studies show that genes in which the promoters are occupied by multiple factors, such as Oct4, Sox2, Nanog and Klf4, are generally active in ESCs; in contrast, genes that bind fewer or single factors tend to be inactive or repressed (Shi and Jin, 2010).

Oct4 is also a repressor that inhibits the expression of lineage specific genes during the differentiation of pluripotent cells. Oct 4 forms a repressive complex with and inhibits the transcription of Cdx2, a transcription factor essential for trophectoderm specification. Hypermethyation leads to gene silencing and can be seen in Oct4 expressing cells where the Oct4 recruits a histone $\mathrm{H} 3$ methyl transferase, Eset, to silence the expression of trophoblast-associated genes that restrict extraembryonic trophoblast formation (Shi and Jin, 2010; Zeineddine et al., 2014). Oct4 and other key regulators of pluripotency are restricted to ES cells, cells of the inner cell mass and to cells of the germ line. During cell lineage commitment, Oct4 is rapidly silenced and the appropriate regulators of development lose polycomb $(\mathrm{PcG})$ mediated repression and are activated. Retinoic acid, a major repressor of Oct4 gene expression has been linked strongly with neural differentiation. Other nuclear repressors like ARP-1, COUP-TF1 and GCNF has been identified that are induced in differentiating cells and are required for complete silencing of Oct4. Epigenetic changes at the Oct4 locus are mostly H3K4me 3 and H3K7 and H3K9 acetylation that leads to the repression of Oct4 gene expression. Further DNA modifications such as CpG DNA methylation also results in silencing of Oct4. As the embryonic stem cells differentiate, an inactive heterochromatin like state is adopted at the Oct4 gene loci (Jaenisch and Young, 2008). A central role for Nanog in the genetic hierarchy that defines ESC identity was earlier established. Nanog expression was upregulated in pluripotent mouse and human ES and embryonic gonadal (EG) cells. Early during ESC differentiation, a down regulation of the Nanog gene was consistent with a loss of pluripotency. Several attributes of Nanog point to its significance as one of the key factors in maintaining stem cell pluripotency. It can be seen that Nanog is required in the maintenance of pluripotency in mouse epiblasts and ESC. Nanog is active independent of LIF/Stat3 in the pluripotent embryonic stem cells. Absence of Nanog expression in ICM cells fails a proper epiblast development and didn't further develop into the extra embryonic endoderm lineage. Thus, a central role for Nanog as a transcription factor that defines ESC pluripotency can be established (Mitsui et al.,2003; Chambers et al.,2003, Zhao et al.,2012). 
Nanog is normally limiting in ES cell cultures and the Oct4/Sox2/Nanog trio of transcription factors is subject to a component of negative regulation. An induced constitutive expression of Nanog prevents ES cell differentiation and initiates self- renewal abilities even when there is an active FGF/Erk signaling. Expression levels of Nanog are highly variable in ES cells contrasting with the apparent homogeneity of Oct4 and Sox2. ES cells in which both alleles of Nanog have been deleted can remain undifferentiated and pluripotent. Other pluripotency genes and many putative targets of Nanog identified by genome location analyses continue to be expressed in ES cells lacking Nanog. These findings challenge the dogma that Nanog acts in partnership with Oct4 and Sox2 in housekeeping transcriptional maintenance of pluripotency. However, cells lacking Nanog, whether by endogenous downregulation or gene deletion, do have a greatly increased tendency to differentiate. In vivo deletion and cell fusion studies have demonstrated that Nanog functions in establishing de novo the unrestricted states of pluripotency. In ES cell cultures, therefore, Nanog may reverse the effects of Erk signaling or of secondary inducers, resetting the ground state. Disruption of Oct4 or Nanog consequently lead to the improper differentiation of ICM and ES cells to trophectoderm and extra-embryonic endoderm respectively (Chambers et al., 2003; Mitsui et al.,2003, Chambers et al.,2007).

\section{Distinct cellular transitions and hierarchies within the ES cell system.}

Oct4, Sox2, and Nanog are central to the transcriptional regulatory hierarchy that specifies ES cell identity because of their unique expression patterns and their essential roles during early development. Though Nanog, Oct4, and Sox 2 constitute the core regulators of ESC pluripotency, they regulate distinct cell fates depending on the soluble factors and signals present. At high concentrations of Oct4, absence of BMP4 results in self-renewal and presence of BMP induces mesoderm differentiation of embryonic stem cells. At low levels of Oct4, absence of BMP4 induces embryonic ectoderm differentiation but specify extraembryonic lineages when BMP4 is present. Nanog is shown to repress embryonic ectoderm differentiation and Sox2 repress mesoderm differentiation. Thus, it can be said that each core factor controls specific cell fates. The embryonic cells integrate signals in their environment and likewise decide whether to remain pluripotent or to differentiate into progenitors of different lineages (Thomson et al.,2011; Wang et al.,2012). ES cells exhibit transcriptional activity for many genes associated with lineage commitment and differentiation. These genes fluctuate in their expression patterns and levels. Therefore, the conventional thinking of ES cell cultures being homogeneous can no longer be considered. Rather, it can be said that the pluripotent ES cells comprise a coalition of transitory transcriptional settings. Pluripotency markers, such as the intracellular transcription factors Oct4, Nanog, enzyme alkaline phosphatase (AP) and the cell surface markers SSEA3, SSEA 4, TRA-1-81, TRA-1-60, and CD24 are widely expressed in pluripotent cells. In culture conditions, however, these molecules didn't exhibit similar expression kinetics and showed a variation of one over another in their expressions patterns (Tai et al.,2005; Shi and Jin 2010; Remirez et al.,2011; Frum et al.,2013; Zeineddine et al.,2014).

Oct4 gene and Oct4 protein are expressed in several adult pluripotent stem cells, but not in normal differentiated daughters of these stem cells. Oct 4 was the first marker to be lost during differentiation, followed by SSEA3 and TRA-1-60 and their expression was completely abolished as differentiation continued. By contrast, AP activity, CD24, and NANOG proteins were still detectable in the differentiating cells. In addition, differentiation markers were coexpressed with pluripotency markers before the latter begin to disappear. These results suggest that Oct4, SSEA3 and TRA are sensitive, specific and quantitative indicators of undifferentiated human embryonic stem cells and are lost upon the induction of differentiation signals. In the embryo, Nanog expression is also dynamic as it is downregulated at the time of implantation and re-emerges in the posterior egg cylinder. It can be inferred that varying levels of Nanog may impart functional heterogeneity in ES cells (Tai $e t$ al., 2005; Chambers et al.,2007; Silva and Smith, 2008; Berg et al.,2010; Ramiraz et al.,2011).

The core transcriptional regulatory factors exhibit tranactivational activity whereby they can bind to other actively transcribed protein coding and miRNA genes in ESCs. As transactivators, Oct4 and Nanog bind and recruit multiple coactivators, to the regulatory elements of the genes that are to be expressed. The coactivators are often multifunctional and can activate transcription through genetic and epigenetic mechanisms. It is also seen that there is formation of DNA looping through different activator subunits thereby long-distance interactions between enhancers and core promoters is made possible. Like the activators, a dose dependent expansion of the transcriptional circutory of coactivator complexes, their protein levels and subunit compositions are noticeable in different developmental stages. These coactivator-activator complexes configure to form the critical nodes in various signal transduction pathways and can serve as molecular switches by integrating intrinsic and extrinsic cues, thereby coupling various genetic networks with specific cellular responses required for the specified gene expressions (Young, 2011; Fong et al.,2011; Hammachi et al.,2011).

The ESC regulatory circuit down regulates as the cell prepares for differentiation. There is a loss of Oct4 and Nanog expressions. As Oct4 is downregulated, transcription of lineage-specific genes is initiated. There is also lineage specific cofactor interactions that are now not under the regulatory control of Oct4. As the ESCs are in 
the differentiated state, Oct4 is silenced by epigenetic modifications and other pluripotent factors like Nanog is reported to undergo caspase mediated proteolytic destruction (Young, 2011).

Silencing of developmental regulators that are directing the lineage commitment and cellular differentiation is an essential component required for pluripotency. Oct4, Sox2, and Nanog in ES cells cooccupy the promoters of these developmental transcription factors moulding them silent in the pluripotent ES cells. MyoD, a transcription factor for muscle lineage is one such gene under suppression by Oct4, Sox2, and Nanog. Another group of proteins, namely the polycomb group (PcG) proteins, major epigenetic regulators that facilitate maintenance of cell state through gene silencing are targets of Oct4, Sox2, and Nanog genetic circutory. The PcG proteins form multiple polycomb repressive complexes (PRCs) that forms a part of epigenetic gene silencing mechanism by mediating histone H3 lysine-27 (H3K27) methylation. PRC1 also contains a histone ubiquitin ligase, Ring1b, whose activity appears likely to contribute to silencing in ES cells (Jaenisch and Young, 2008; Hammachi et al., 2011).

Gene sequences are transcribed under the control of cis and trans acting elements including the transcriptional factors. They enable the proper binding of RNA polymerase to the regulatory sequences or recruit a cyclin-dependent enzyme that phosphorylates the polymerase. Phosphorylation stabilizes the enzyme binding and completes full transcription. Oct4, Sox2, and Nanog might exert their effects by regulating RNA polymerase II during the process of gene expression. Polycomb complexes and other cofactors may hold RNA polymerase at key regulatory sequence of differentiation promoting genes in pluripotent cells and in lineage cells. Concurrently a full length transcription is not completed due to the PcG mediated sequence binding at the regulatory sequences and thereby causing repression of gene expression. In differentiated cells, PcG proteins and its further H3K27 methylation are lost allowing the transcription apparatus to fully transcribe these genes. (Jaenisch and Young, 2008; Young, 2011).

Pluripotent embryonic stem cells can be maintained in an undifferentiated state in culture, but once culture conditions are withdrawn, they start to rapidly differentiate. Certain extracellular signals have been studied and reported to contribute to the maintenance of ES cell pluripotency. A few might also take part in the differentiation process. The core factors Oct 4 , Sox 2 and Nanog interacted transactivationally with the transcription factors associated with the LIF, Wnt, and BMP4 signaling pathways. The signaling factors were shown to co-occupy the same binding regions as the enhancers that were required to maintain the pluripotency or repress the differentiation potential (Zeineddine et al., 2014).

Leukemia inhibitory factor (LIF) is one of the major signaling molecules expressed in the pluripotent murine ES cells. LIF/Stat3 promotes ES cell growth and viability. In vitro stimulation of the Stat3 signaling pathway by the cytokine LIF and blocking the expression of differentiation (ID) proteins by serum inhibitory factors or bone morphogenetic proteins (BMPs) leads to activation of Erk signaling. The LIF is known to be critical for the maintenance of pluripotency in murine ES cells while it seems not an essential pathway in $\mathrm{hES}$ cells. In mES cells, LIF via activation of JAK1/ STAT3 pathway is an essential cytokine that prevents differentiation into different lineages. Stat 3 activates the expression of a significant number of pluripotencyrelated genes in ES cells. Some of these genes are also regulated by Oct4 extending the circutory that controls the pluripotency of embryonic stem cells. Oct4 and Stat3 bind to the promoter region of embryonic ectoderm development gene (Eed gene) which encodes for a core component of Polycomb repressive complex 2. Klf4 (Krüppel-like transcription factor 4) is a direct downstream target of Oct4 and Stat 3 and is expressed to maintain the ES cell self-renewal and pluripotency. It seems that the cooperation between the intrinsic Oct4 network and extrinsic LIF pathway is crucial to regulate ES cell self-renewal and pluripotency (Young, 2011; Ura et al.,2011; Zeineddine et al.,2014).

FGF4 is under the regulatory control of Oct4 and Sox2 and active expression of FGF4 acts as an autoinductive stimulus that propels ES cells toward lineage specification. Inhibition of the FGF4/Erk axis by genetic disruption or pharmacological inhibitors induces self-renewal without involving Stat3 signaling. Therefore, ES self-renewal appears to be uncommitted until the appropriate molecular circuitry signals are activated. FGF4/Erk signal does not specify any particular lineage but might render ES cells responsive to further inductive signals. This was evidenced in ES cells or epiblast lacking FGF4 or deficient in downstream mitogen activated protein kinase Erk1/2 signaling, where a general impairment of lineage differentiation was indicated. ERK activity can be retained within the culture by selective inhibition of glycogen synthase kinase-3 (GSK3) through canonical Wnt signaling mediated by intracellular $\beta$-catenin or by non-canonical Wnt signaling. Wnt3a reinforces the suppression of neural commitment and may increase the probability of a self-renewal division. However, Wnt has only minor effects on ES cell growth and viability and does not fully reproduce the effects of GSK3 inhibition. In ES cells, silencing and activation of the Notch and BMP4 pathways is shown to promote self renewal and differentiation respectively. With Notch pathway, there is neural differentiation in both human and mouse embryonic stem cells. BMP4, on the other hand most favourably induces differentiation into other cell types (Kunath et al., 2007; Anton et al.,2007; Jaenisch and Young, 2008). 


\section{Is epigenetic mechanism a major determinant of stem cell pluripotency?}

Gene expression can be regulated by epigenetic modifications. The components involved in the transcriptional control by epigenetic changes are collectively referred as chromatin regulators. Majority of the chromatin regulators are concerned with DNA condensation, histone modifications, chromatin-remodeling and methylations. The epigenetic mechanisms in ES cells are characterized by coexistence of both activating and silencing chromatin modifications at multiple loci. Activating modification and Repressive may be integral to pluripotency by providing a mechanism for repressing gene expression without permanent silencing. In stem cells, their role in maintaining pluripotency is subjective to a partial contribution as loss in epigenetic mechanism doesn't alter the pluripotency status of the stem cells. In fact the pluripotent state was altered only when an irreversible change in developmental potential occurred as seen in Oct4 or Sox 2 deletion. It can be inferred that epigenetic mechanisms do contribute but to a lesser extent than Oct 4 or Sox2. The main event of epigenetic changes is histone modification that was significantly found to repress genes encoding lineagespecific developmental cues. Silencing of differentiation specific genes in the ground state is thought to be a part of DNA methylation as established by the presence of DNA methylating enzymes such as Dnmt1, 2, 3a, 3b, and 31 in ES cells. Most of CpG dinucleotides are methylated in the ESCs though it was not considered as essential for the embryonic state attainment (Niwa, 2007; Young, 2011).

\section{Cellular reprogramming into ESC-like states}

In iPSCs, the extrinsic addition of these factors as gene cocktails might activate the ground state in which the newly dividing cells in culture might retain their pluripotent nature rather than differentiating into specific lineages. It can also be considered that the induced expression through the addition of exogenous factors during reprogramming might activate transcription of the endogenous Oct4, Sox2, and Nanog genes and thereby initiate the ground status. Addition of some more factors in the reprogramming cocktails, such as c-Myc, migh facilitate activation of the ground state circutory by stimulating gene expression and proliferation more generally (Jaenisch and Young, 2008; Rahl et al.,2010; Young, 2011).

\section{Conclusion}

The most important regulatory aspects in ESCs seems to be constituted by a small number of "core" transcription factors acting in concert with other transcription factors, some of which are also a part of several signaling pathways. Studies implicate transcription factors Oct4, Sox2, and Nanog in maintaining the stem cell pluripotency in vivo and in vitro. Yet remarkably Oct 4 and Sox 2 also direct production of destabilizing signals that commits ES cells into specific lineages. A balance between the pluripotency and differentiation might be essential for an uncommitted ground state which can be tapped for the authentic ES cells for cell replacement therapies.

As in any differentiation process, the stem cells under the control of factors that can act synergistically or antagonistically have to make the final call of whether to remain in the ground state or in the differentiated state. In the differentiation of tissue-specific lineages, regulatory factors often operate in both positive and negative fashions to refine the ultimate developmental decision. Through feed forward and auto regulatory loops, Oct4, Sox 2 and Nanog promote expression of other pluripotency/self-renewal genes as well as themselves, while simultaneously preventing expression of differentiation-promoting genes. Though such loops act together in stem cells, studies is still needed to identify the other might be key players in the circuits. How are these key players regulated by the stem cell niche cues, which are mostly constituted by extracellular signals? Consistency in maintaining the pluripotency is lost when one of the key players is down regulated. More insights are required for a proper understanding of dedifferentiation and induced adult pluripotent stem cells for their use as translational medicines.

\section{References}

[1]. Anton R, Kestler HA and Kuhl M. (2007). $\beta$-Catenin signaling contributes to stemness and regulates early differentiation in murine embryonic stem cells. FEBS Lett. 581, 5247-5254.

[2]. Boyer LA, Lee TI, Cole MF, Johnstone SE, Levine SS, Zucker JP, Guenther MG, Kumar RM, Murray HL, Jenner RG, Gifford DK, Melton DA, Jaenisch R and Young RA. (2005). Core transcriptional regulatory circuitry in human embryonic stem cells. Cell. 122, 947-956.

[3]. Chambers I, Colby D, Robertson M, Nichols J Lee, Tweedie S and Smith, A. (2003). Functional Expression Cloning of Nanog, a Pluripotency Sustaining Factor in Embryonic Stem Cells. Cell. 113, 643-655.

[4]. Chambers I, Silva J, Colby D, Nichols J, Nijmeijer B, Robertson M, Vrana J, Jones K, Grotewold L and Smith A. (2007). Nanog safeguards pluripotency and mediates germline development. Nature. 450, 1230-1234

[5]. Fong YW, Inouye C, Yamaguchi T, Cattoglio C, Grubisic I and Tjian R. (2011). A DNA repair complex functions as an Oct4/Sox2 coactivator in embryonic stem cells. Cell. 147, 120-131.

[6]. Frum T, Halbisen MA, Wang C, Amiri H, Robson P and Ralston A. (2013). Oct4 Cell-Autonomously Promotes Primitive Endoderm Development in the Mouse Blastocyst. Developmental Cell. 25, 610-622. 
[7]. Hammachi F, Morrison GM, Sharov AA, Livigni A, Narayan S, Papapetrou EP, O’Malley J, Kaji K, Ko MSH, Ptashne M and Brickman JM. (2012). Transcriptional Activation by Oct4 Is Sufficient for the Maintenance and Induction of Pluripotency. Cell Reports. 1, 99-109.

[8]. Han DW, Tapia N, Hermann A, Hemmer K, Hoing S, Arauzo-Bravo MJ, Zaehres H, Wu G, Frank S, Moritz S, Greber B, Yang JH, Lee HT, Schwamborn JC, Storch A and Scholer HR. (2012). Direct Reprogramming of Fibroblasts into Neural Stem Cells by Defined Factors. Cell Stem Cell. 10, 465-472.

[9]. Huang S, Guo YP, May G and Enver T. (2007). Bifurcation dynamics in lineage-commitment in bipotent progenitor cells. Dev. Biol. 305, 695-713.

[10]. Jaenisch R and Young R. (2008). Stem Cells, the molecular circuitry of pluripotency and nuclear reprogramming. (2008). Cell. 132, 567-582.

[11]. Kerr IC, Hill CM, Blumenthal PD and Gearhart JD. (2008). Expression of pluripotent stem cell markers in the human fetal testis. Stem Cells. 26, 412-421.

[12]. Kunath T, Saba-El-Leil MK, Almousailleakh M, Wray J, Meloche S and Smith A. (2007). FGF stimulation of the Erk1/2 signalling cascade triggers transition of pluripotent embryonic stem cells from self-renewal to lineage commitment. Development. 134, 28952902.

[13]. Lee J, Yeorim GO, Kang I, Han YM and Kim J. (2010). Oct-4 controls cell-cycle progression of embryonic stem cells. Biochem. J. 426, 171-181.

[14]. Mitsui K, Tokuzawa Y, Itoh H, Segawa K, Murakami M, Takahashi K, Maruyama M, Maeda M and Yamanaka S. (2003). The homeoprotein Nanog is required for maintenance of pluripotency in mouse epiblast and ES cells. Cell 113, 631-642.

[15]. Nichols J and Smith A. (2012). Pluripotency in the Embryo and in Culture. Cold Spring Harb Perspect Biol. $4:$ 1-14.

[16]. Niwa, H. (2007). How is pluripotency determined and maintained? Development. 134, 635-646.

[17]. Pan GJ, Chang ZY, Scher HR and Pei D. (2002). Stem cell pluripotency and transcription factor Oct4. Cell Research. 12, $321-329$.

[18]. Pierantozzi E, Gava B, Manini I, Roviello F, Marotta G, Chiavarelli M and Sorrentino V. (2010). Pluripotency regulators in human mesenchymal stem cells: Expression of Nanog but not of Oct4 and Sox2. Stem Cells and Development. 20, 915-923.

[19]. Rahl PB1, Lin CY, Seila AC, Flynn RA, McCuine S, Burge CB, Sharp PA, Young RA. (2010). c-Myc regulates transcriptional pause release. Cell. 41, 432-45.

[20]. Ramirez JM, Gerbal-Chaloin S, Milhavet O, Qiang B, Becker F, Assou S, Lemaitre JM, Hamamah S and Vos JD. (2011). Brief Report: Benchmarking human pluripotent stem cell markers during differentiation into the three germ layers unveils a striking heterogeneity: all markers are not equal. Stem Cells. 29, 1469-1474.

[21]. Shi G and Jin Y. (2010). Role of Oct4 in maintaining and regaining stem cell pluripotency. Stem Cell Research \& Therapy. 1:39.

[22]. Silva J and Smith A. (2008). Capturing Pluripotency. Cell, 132, 532-536.

[23]. Tai MH, Chang CC, Olson LK and Trosko JE. (2005). Oct4 expression in adult human stem cells: evidence in support of the stem cell theory of carcinogenesis. Carcinogenesis. 26, 495-502.

[24]. Takahashi K and Yamanaka S. (2006). Induction of pluripotent stem cells from mouse embryonic and adult fibroblast cultures by defined factors. Cell 126, 663-676.

[25]. Thomson M, Liu SJ, Zou LN, Smith Z, Meissner A and Ramanathan S. (2011). Pluripotency factors in embryonic stem cells regulate differentiation into germ layers. Cell 145, 875-889.

[26]. Tsai CC, Su PF, Huang YF, Yew TL and Hung SC. (2012). Oct4 and Nanog directly regulate Dnmt1 to maintain self renewal and undifferentiated state in mesenchymal stem cells. (2012). Molecular Cell. 47, 169-182.

[27]. Ura H, Murakami K, Akagi T, Kinoshita K, Yamaguchi S, Masui S, Niwa H, Koide H and Yokota T. (2011). Eed/Sox2 regulatory loop controls ES cell self renewal through histone methylation and acetylation. EMBO J. 30, 2190-204.

[28]. Van den Berg DLC, Snoek T, Mullin NP, Yates A, Bezstarosti K, Demmers J, Chambers I and Poot RA. (2010). An oct4-centered protein interaction network in embryonic stem cells. Cell Stem Cell. 6, 369-381.

[29]. Wang Z, Oron E, Nelson B, Razis S and Ivanova N. Distinct lineage specification roles for Nanog, Oct4 and Sox 2 in human embryonic stem cells. (2012). Cell Stem Cell. 10, 440-454.

[30]. Webster JD, Yuzbasiyan-Gurkan V, Trosko JE, Chang CC and Kiupel M. (2007). Expression of the embryonic transcription factor oct4 in canine neoplasms: a potential marker for stem cell subpopulations in neoplasia. Vet Pathol. 44, 893-900.

[31]. Yamanaka S and Blau HM. (2010). Nuclear reprogramming to a pluripotent state by three approaches. Nature. 465, 704-712.

[32]. Young RA. (2011). Control of the embryonic stem cell state. Cell. 144, 940-54.

[33]. Zeineddine D, Hammoud AA, Mortada M and Boeuf H. (2014). The Oct4 protein: more than a magic stemness marker. Am J Stem Cells. 3, 74-82.

[34]. Zhao W, Ji X, Zhang F, Li L and Ma L. (2012). Embryonic Stem Cell. Markers. Molecules. 17, 6196-6236.

[35]. Zhu Z and Huangfu D. (2013). Human pluripotent stem cells: an emerging model in developmental biology. Development. 140, $705-717$.

\section{Figures}

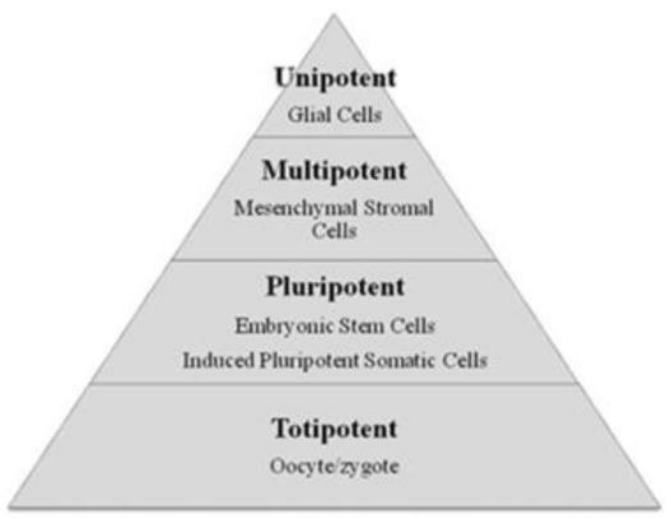

Fig 1: Stem cells and their developmental potential 


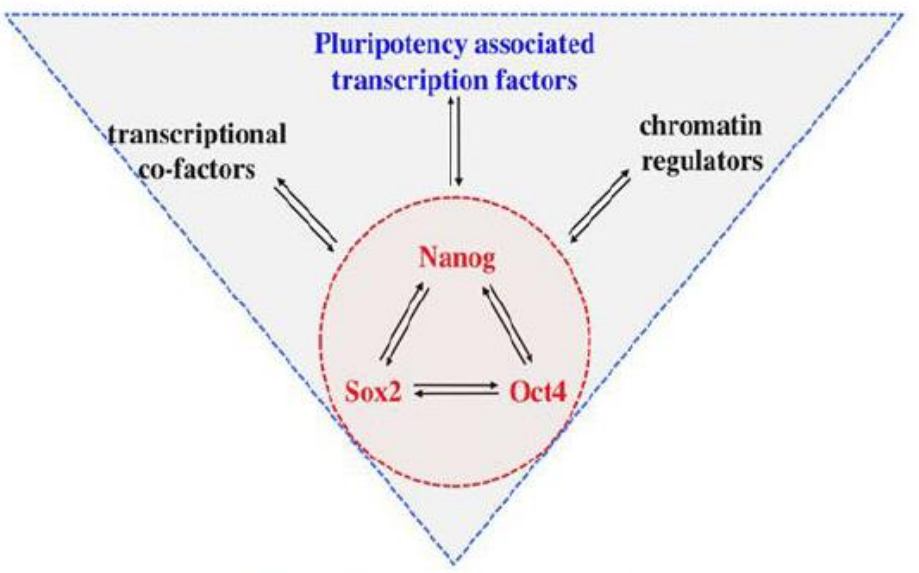

Fig. 2: ESC transcriptional regulatory circuitry 